\title{
Discussion: Mechanical properties of hybrid fibre-reinforced concrete - analytical modelling and experimental behaviour
}

\section{Aref Abadel}

MMB Chair for Research and Studies in Strengthening and Rehabilitation of Structures, Department of Civil Engineering, King Saud University, Riyadh, Saudi Arabia

\section{Husain Abbas}

MMB Chair for Research and Studies in Strengthening and Rehabilitation of Structures, Department of Civil Engineering, King Saud University, Riyadh, Saudi Arabia

\section{Tarek Almusallam}

MMB Chair for Research and Studies in Strengthening and Rehabilitation of Structures, Department of Civil Engineering, King Saud University, Riyadh, Saudi Arabia

\section{Yousef Al-Salloum}

MMB Chair for Research and Studies in Strengthening and Rehabilitation of Structures, Department of Civil Engineering, King Saud University, Riyadh, Saudi Arabia

\section{Nadeem Siddiqui}

MMB Chair for Research and Studies in Strengthening and Rehabilitation of Structures, Department of Civil Engineering, King Saud University, Riyadh, Saudi Arabia

\section{Mohammed A. Shubaili}

Civil Engineering Department, Jazan University, Jazan, Saudi Arabia

Hossam El-Din M. Sallam

Materials Engineering Department, Zagazig University, Zagazig, Egypt

\section{Contribution by M. A. Shubaili and H. El-Din M. Sallam}

The discussers appreciate the effort made by the authors in proposing an analytical model to predict the mechanical behaviour of hybrid fibre-reinforced concrete (HyFRC or HFRC) taking into consideration the synergy concept (Abadel et al., 2016). However, their experimental results contradict this concept. For example, all the properties of mix M4 ( $0 \cdot 2 \%$ PPF + $1 \cdot 2 \% \mathrm{SF}$ ) are lower than those of steel fibre-reinforced concrete (SFRC) having only $1 \cdot 2 \% \mathrm{SF}$, mix $\mathrm{M} 1$, that is, negative synergy. Generally, for the same fibre volume fraction $\left(F_{\mathrm{Vf}}\right)$, all the properties of HyFRC mixes are lower than those of SFRC mixes, as shown in Figure 10. Furthermore, the modulus of rupture, splitting tensile strength and flexural toughness of SFRC with a lower $F_{\mathrm{Vf}}$, mix 1 , are greater than those of mix 3 (SFRC with a higher $F_{\mathrm{Vf}}$ ). Although the authors mentioned that 'The fibres were added to plain concrete in parts to prevent fibre balling and to ensure the homogeneity of the concrete mixture. The mixing was done for about $3 \mathrm{~min}$ to ensure the proper distribution of fibres in the concrete mass', they did not show the readers any validation for this, such as images of the fracture surfaces of the different types of specimens showing the uniform dispersion of the three different types of fibres, or any statistical analysis of the experimental results, such as the standard deviation or the coefficient of variation. Casting and compaction affect the fibre orientation throughout the specimen and could potentially lead to fibre segregation. Furthermore, different types of fibres require different optimum ranges of rheology of the cementitious mortar to achieve good fibre dispersion. This can lead to a significant challenge in processing hybrid fibres in the same matrix; see, for example, Abou El-Mal et al. (2015).

\section{Authors' reply}

The authors would like to thank the discussers for their interest in this paper and their insightful comments on the work. The authors' responses to the individual comments are given below under two main headings.

\section{Synergy in HFRC mixes}

In the study under discussion, the synergy in different HFRC mixes was only observed to some extent for mix M7, as mentioned in the section of the original paper entitled 'Discussion of test results'. As the main objective of adding fibres to concrete is to improve the tensile characteristics of the concrete, such as modulus of rupture, splitting tensile strength and direct tensile strength, these properties of HFRC mixes having 1.4\% volume fraction of fibres are compared with mix M3 (containing only steel fibres) and shown in Figure 11. It is to be noted here that the tensile characteristics presented in the figure are in terms of the compressive strength of concrete, that is, the ratio of tensile characteristics to the compressive strength. The figure shows the presence of synergy in mix M7. As the ratio of M7 to M3 is slightly greater than unity, the synergy in mix M7 was not strongly claimed in the paper. Other researchers (e.g. Banthia and Gupta, 2004) have also reported synergy effects in some of their mixes, but not in all of the mixes. 


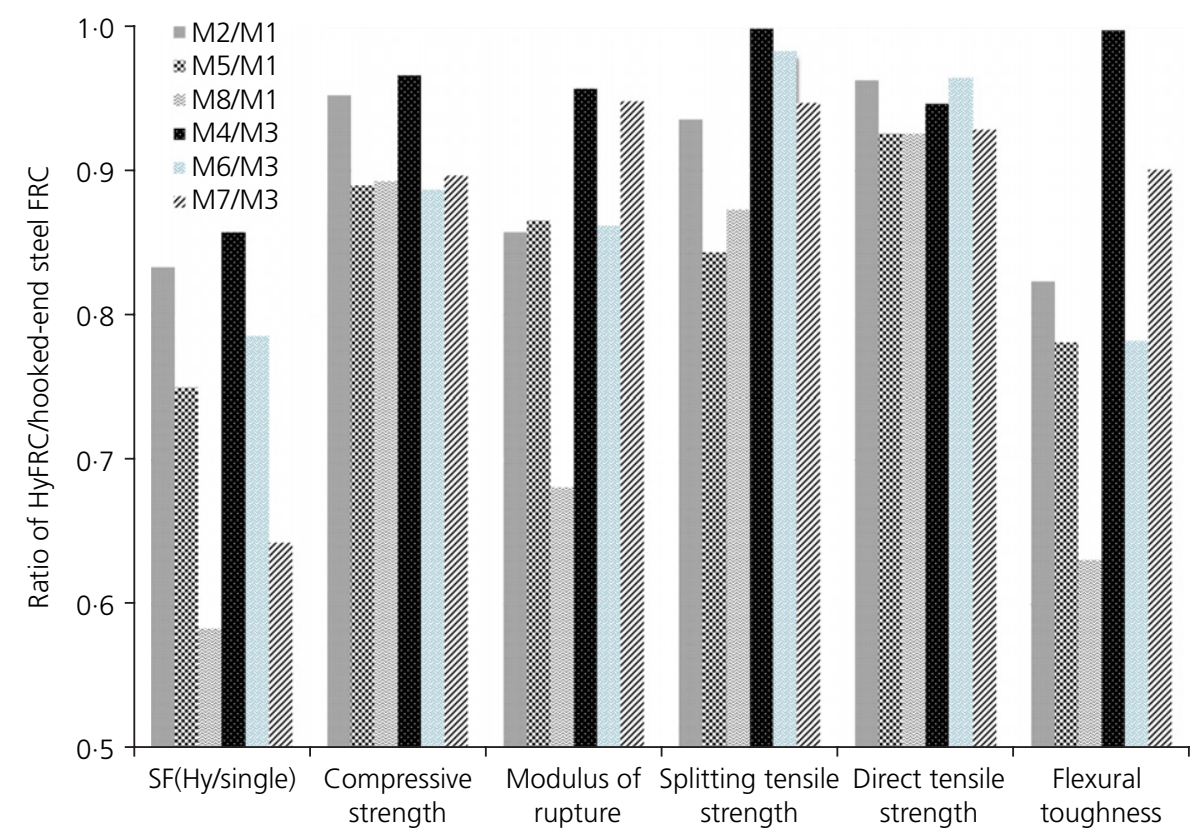

Figure 10. A comparison of various characteristics of HyFRC mixes with steel FRC

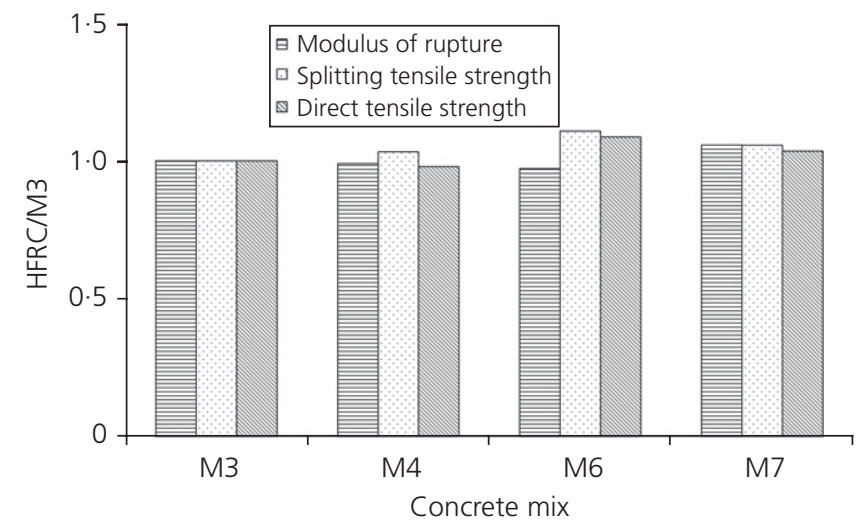

Figure 11. A comparison of tensile characteristics of HFRC mixes containing $1.4 \%$ total volume fraction of fibres (note: the tensile characteristic is taken as the ratio of the tensile strength parameter to the compressive strength)

Another aspect of positive synergy, although not presented in the paper, is that the presence of plastic fibres improves the fire endurance of concrete by providing a route along the molten fibres for the release of gases to the outside atmosphere, thereby avoiding explosive bursting of concrete.

\section{Dispersion of fibres}

Regarding the comparison of M1 and M3, the increase in the percentage of steel fibres from $1 \cdot 2$ to $1 \cdot 4 \%$ caused a small change in the mechanical properties, with the compressive strength and direct tensile strength increasing, but modulus of rupture and split tensile strength being marginally reduced. The authors are aware of the issues of fibre dispersion caused by fibre balling/agglomeration and near-surface effects. The mixing of fibres in concrete is a great challenge and, for the hybrid fibres, which are a mixture of stiff (steel) and flexible fibres (polypropylene and Kevlar), the mixing becomes more challenging. The reinforcing ability of the fibres depends on the distribution and orientation of fibres in the concrete. The factors responsible for poor dispersion of fibres and consequent flaws in HFRC mixes are listed below.

- The shapes of the fibres are more elongated as compared to coarse aggregates, so this may cause interlocking.

- Stiff fibres of steel alter the intergranular arrangement in concrete, whereas the flexible fibres may not affect it to that extent and may bend themselves, thereby increasing the porosity of the concrete. These phenomena are more pronounced when aggregates of large size are used in concrete.

- Hooked ends of steel fibres may cause entanglement and increase the friction between fibres and aggregates.

- The mixing of long fibres also affects the compactability of concrete.

- There is a change in orientation and bending of both stiff and flexible fibres close to the mould boundaries.

- There can be excessive mixing, especially when coarse aggregates of large size are used, which may cause fibre balling.

Researchers (Li and Li, 2013; Ozyurt et al., 2007) have used different combinations of plasticising agents, viscosity 
modifiers and vibration duration for controlling fibre dispersion, but with limited success. Controlling fibre dispersion is generally difficult and new methods are required. Convenient methods are also needed for evaluation of fibre dispersion because most methods currently used are either approximate, destructive and/or time consuming. The discussers' idea of monitoring fibres through imaging of the fracture surface is rather crude and time consuming, as it will show only the location of fibres, and only those at the fracture surface. For monitoring fibre orientation, segregation and clumping, alternating current-impedance spectroscopy (AC-IS) has been used (Ozyurt et al., 2006, 2007), but this method can only be used for conductive fibres. An alternative method to AC-IS is image analysis, whereby the fibres of a cross-section

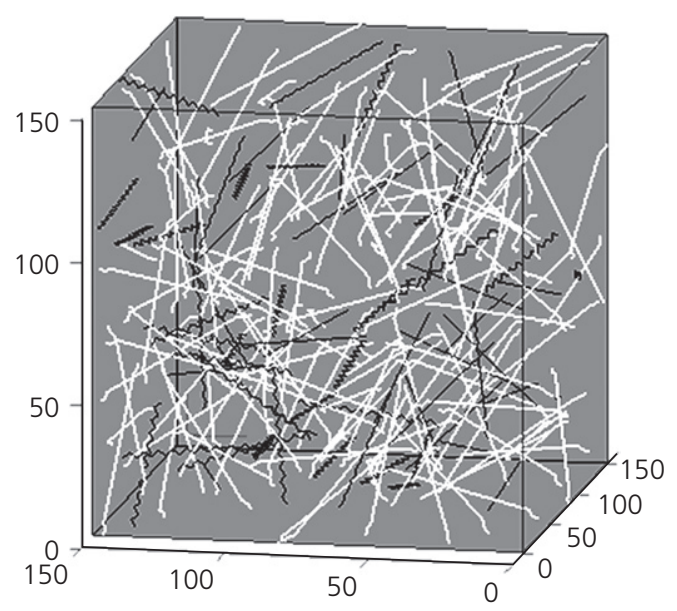

(a)

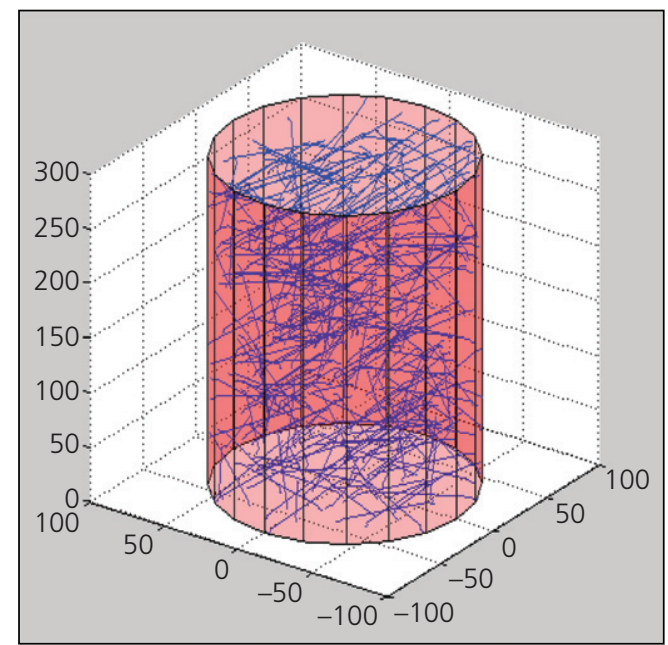

(b)

Figure 12. Distribution of $0 \cdot 18 \%$ volume fraction of fibres in: (a) $150 \mathrm{~mm}$ cube containing a mixture of hooked steel (white), crimped polypropylene (black) and straight Kevlar fibres (white); (b) standard cylinder $150 \times 300 \mathrm{~mm}$ containing hooked-end steel fibres (note: all dimensions shown in $\mathrm{mm}$ ) are counted using a probe sensitive to the magnetic properties of the steel fibres (Ferrara et al., 2012; Ozyurt et al., 2006, 2007; Stroeven and $\mathrm{Hu}, 2006$ ). Recently some researchers (Le et al., 2008; Suuronen et al., 2013; Wuest et al., 2009) have employed X-ray tomography to determine the distribution and orientation of metallic fibres in hardened concrete samples. These issues, including those suggested by the discussers, were not within the scope of the paper. Instead of showing the scattering through statistical values, it is shown graphically in Figures 8(a) and 8(b) for some of the properties of HFRC. Moreover, authors are currently working in this field and trying to relate the mechanical properties of HFRC to the above-mentioned issues of fibre distribution and orientation. Figure 12 shows the numerical models with simulated fibre distribution and orientation. These models will be used for finite-element analysis of cubes and cylinders employed for determining the mechanical properties of HFRC. To show different fibres distinctly, a low magnitude of the total volume fraction of fibres $(0 \cdot 18 \%)$ is considered in this figure. The results of these analyses will be published in the near future.

It is also worth mentioning that the above experimental issues, including those mentioned by the discussers, were not given more emphasis in order to limit the size of the paper and provide enough space for presenting and validating the analytical modelling, which was a major component of the paper.

\section{REFERENCES}

Abadel A, Abbas H, Almusallam T, Al-Salloum Y and Siddiqi N (2016) Mechanical properties of hybrid fibre-reinforced concrete - analytical modelling and experimental behaviour. Magazine of Concrete Research 68(16): 823-843, http://dx.doi.org/10.1680/jmacr.15.00276.

Abou El-Mal HSS, Sherbini AS and Sallam HEM (2015) Mode II fracture toughness of hybrid FRCs. International Journal of Concrete Structures and Materials 9(4): 475-486.

Banthia N and Gupta R (2004) Hybrid fiber reinforced concrete (HyFRC): fiber synergy in high strength matrices. Materials and Structures 37(10): 707-716.

Ferrara L, Faifer M and Toscani S (2012) A magnetic method for non destructive monitoring of fiber dispersion and orientation in steel fiber reinforced cementitious composites - part 1: method calibration. Materials and Structures 45(4): 575-589.

Le TH, Dumont P, Orge'as L et al. (2008) X-ray phase contrast microtomography for the analysis of the fibrous microstructure of SMC composites. Composites A 39(1): 91-103.

Li M and Li VC (2013) Rheology, fiber dispersion, and robust properties of engineered cementitious composites. Materials and Structures 46(3): 405-420.

Ozyurt N, Mason TO and Shah SP (2006) Non-destructive monitoring of fiber dispersion in FRCs using AC-impedance spectroscopy. In Measuring, Monitoring 
and Modeling Concrete Properties (Konsta-Gdoutos MS (ed.)). Springer, Dordrecht, The Netherlands, pp. 285-290. Ozyurt N, Mason TO and Shah SP (2007) Correlation of fiber dispersion, rheology and mechanical performance of FRCs. Cement and Concrete Composites 29(2): 70-79.

Stroeven P and Hu J (2006) Review paper - stereology: historical perspective and applicability to concrete technology. Materials and Structures 39(1): 127-135.
Suuronen JP, Kallonen A, Eik M et al. (2013) Analysis of short fibres orientation in steel fibre-reinforced concrete (SFRC) by X-ray tomography. Journal of Material Science 48(3): $1358-1367$.

Wuest J, Denarie E, Bruhwiler E et al. (2009) Tomography analysis of fiber distribution and orientation in ultra high-performance fiber-reinforced composites with high-fiber dosages. Experimental Techniques 33(5): 50. 